\title{
Исследование подвижности носителей заряда в слоях нанокристаллов PBS методом полевого транзистора
}

\author{
(ㄱ П.С. Парфенов, Н.В. Бухряков, Д.А. Онищук, А.А. Бабаев, А.В. Соколова, А.П. Литвин \\ Университет ИТМО, Центр „Информационные оптические технологии“, \\ 197101 Санкт-Петербург, Россия \\ E-mail: qrspeter@gmail.com
}

Поступила в Редакцию 1 сентября 2021 г.

В окончательной редакции 15 октября 2021 г.

Принята к публикации 15 октября 2021 г.

\begin{abstract}
Методом полевого транзистора исследуется подвижность носителей зарядов в слоях нанокристаллов сульфида свинца с лигандами тетрабутиламмония иодида и 1,2-этандитиола, используемых для создания солнечных элементов. Демонстрируется отличие режима работы транзистора на воздухе от работы в условиях инертной среды. Показано, что на открытом воздухе активизируются процессы зарядки нанокристаллов при протекании тока, проанализировано влияние поляризации интерфейса нанокристаллов и изолятора на измерение подвижности. Продемонстрирована разная реакция слоев с лигандами на свет, показывающая существенное окисление поверхности нанокристаллов, обработанных 1,2-этандитиолом.
\end{abstract}

Ключевые слова: солнечные элементы, ловушечные состояния, фотопроводимость.

DOI: 10.21883/FTP.2022.02.51968.9734

\section{1. Введение}

Солнечные элементы (СЭ) на основе нанокристаллов $\mathrm{PbS}$ - популярное направление развития фотовольтаики. Нанокристаллы (HK) $\mathrm{PbS}$ поглощают в широком спектральном диапазоне и характеризуются большим боровским радиусом, позволяющим легко реализовать режим сильного квантового конфайнмента, а значит, и подобрать нужные оптические свойства. Меняя молекулы лигандов, прикрепляющиеся к поверхности $\mathrm{HK} \mathrm{PbS}$, можно изменять расстояние между НК, влияя таким образом на перенос заряда между НК и проводимость внутри слоя соответственно [1], сдвигать положение уровня Ферми и границы запрещенной зоны [2], что нужно для согласования уровней в энергетической структуре устройств, а также изменять тип проводимости слоя, делая доминирующей дырочную или электронную проводимость. Хотя исследования таких устройств проводятся при изготовлении в инертной атмосфере и последующей эксплуатации в инкапсулированном виде, ряд исследований показывают достаточно высокую эффективность подобных устройств и при функционировании в условиях окружающей атмосферы. Установлено, что пребывание на воздухе в течение нескольких дней ведет к улучшению параметров СЭ [3].

На воздухе в слоях $\mathrm{PbS}$ НК происходят процессы, существенно изменяющие их оптические и физические характеристики. Так, из-за окисления поверхности уменьшается эффективный диаметр НК и смещаются максимумы поглощения и люминесценции, а также изменяется время затухания люминесценции [4,5]. Для работы оптоэлектронных устройств более существенно то, что при окислении может измениться диаметр НК, а следовательно, произойти изменение ширины межкристаллического барьера, а также появиться оксидная фаза и дополнительные акцепторные состояния кислорода. Такое появление новых акцепторных состояний может изменить тип проводимости слоя. Так, в нескольких работах показано, что экспозиция на открытом воздухе приводит к изменению типа проводимости НК халькогенидов свинца на дырочную, даже если, благодаря обработке лигандами, она являлась электронной $[1,6]$.

Модели, применяющиеся для описания устройств, работающих на воздухе, в настоящее время полностью основаны на данных, полученных для устройств, изготовленных в инертной среде. В своих работах мы также исходили из такой модели $[7,8]$. В то же время понятно, что полноценное описание функционирования таких устройств, необходимое для оптимизации их работы, требует определения зонной структуры слоев, концентрации носителей, их подвижности, длины свободного пробега и времени жизни - параметров, которые могут существенно меняться под действием окружающей среды. В данной работе мы останавливаемся на изучении подвижности зарядов находящихся на воздухе слоев НК $\mathrm{PbS}$ и выдвигаем предположения относительно отличий в работе СЭ на их основе.

\section{2. Методика эксперимента}

\section{1. Солнечные элементы на основе нанокристаллов PbS}

В данной работе рассматривается функционирование СЭ, основанных на структуре с использованием двух слоев $\mathrm{HK} \mathrm{PbS}$, обработанных лигандами 1,2-этандитиола (1,2-Ethanedithiol, EDT) и тетрабутиламмония иодида (Tetrabutylammonium iodide, TBAI). Такая структура 
ITO/ZnO/PbS-TBAI/PbS-EDT/Au широко распространена, в ней образование электронно-дырочных пар происходит в слое нанокристаллов $\mathrm{PbS}-\mathrm{TBAI}$, разделение заряда происходит на границе слоев нанокристаллов $\mathrm{ZnO} / \mathrm{PbS}-\mathrm{TBAI}$, а слой PbS-EDT выполняет роль транспорта дырок и блокирования электронов. Анализ процессов, происходящих на границе раздела слоев в таких элементах, проводится в работах [9-11].

Для описания процессов в слоях СЭ обычно исследуют мобильность носителей зарядов, их концентрацию, распределение ловушечных состояний, положение уровней Ферми и процессов на интерфейсах слоев. Мы в работе ограничиваемся рассмотрением вопроса мобильности зарядов.

\section{2. Определение подвижности зарядов методом полевого транзистора}

Для измерения подвижности носителей зарядов и определения типа проводимости мы использовали метод полевого транзистора. В этом методе исследуемое вещество образует канал между стоком и истоком, а затвор, отделенный от канала слоем диэлектрика, своим потенциалом отталкивает или притягивает электроны и дырки. Это изменяет концентрацию носителей, а значит, изменяет сопротивление канала и ток между стоком и истоком. Анализируя зависимость тока в канале от потенциала затвора $I_{d s}\left(V_{g s}\right)$, или передаточную характеристику (transfer characteristic, или сток-затворная), можно определить подвижность как электронов, так и дырок. В зависимости от точки на выходной характеристике $I_{d s}\left(V_{d s}\right)$ (output characteristic, или стоковая характеристика), показывающей зависимость тока в канале от напряжения между стоком и истоком при заданном потенциале затвора, выделяют линейный режим и режим насыщения и применяют соответствующие формулы расчета [12].

Значение подвижности носителей определяется из наклона линейного участка переходной характеристки (дырок по $p$-канальной передаточной характеристике при отрицательном смещении затвора и стока, электронов - по $n$-канальной передаточной характеристике при положительном смещении затвора и стока). Для линейного режима подвижность определяется как

$$
\mu_{\text {lin }}=\left.\frac{L}{W C_{o x} V_{d s}} \frac{\partial I_{d s}}{\partial V_{g s}}\right|_{V_{d s}=\text { const }},
$$

где $W$ - ширина канала, $L-$ длина канала, $C_{o x}-$ емкость слоя изолятора на единицу площади (oxide capacitance), $\mu$ - подвижность.

Пороговое напряжение $V_{\text {th }}$ (threshold voltage) соответствует окончанию зоны экспоненциального роста тока на передаточной характеристике, а напряжение начала экспоненциального роста тока называют начальным напряжением (onset voltage) $V_{\text {on }}$, оно соответствует началу образования канала. Величина значений $V_{\text {on }}$ и $V_{\text {th }}$ зависит от разных эффектов (интерфейсные состояния, ловушки и дипольные состояния, примеси [13]). Чаще всего причиной ненулевого напряжения включения являются заряженные ловушечные состояния на интерфейсе с диэлектриком [14]. Существенный сдвиг напряжения включения может быть вызван, например, поляризацией диэлектрика и проявляться в виде гистерезиса [13].

Заметим, что вышеприведенная формула действительна в рамках приближения (Gradual Channel Approximation), предполагающего толщину канала пренебрежимо малой по сравнению с его длиной. В таком случае электрическое поле в канале однородно, а краевыми эффектами можно пренебречь. Поэтому важным моментом при определении мобильности является линейность выбранного участка, поскольку очень часто за линейный участок принимается участок перегиба кривых, что дает завышенное значение мобильности [12]. Если линейного участка ни в одном режиме достичь не удается, то подвижность должна вычисляться исключительно из линейного режима. Это вызвано тем, что режим насыщения более подвержен ошибке, особенно если подвижность зависит от концентрации носителей зарядов, которая меняется вдоль канала и дополнительно может зависеть от заполнения хвостовых состояний запретной зоны (band-tail filling) [15].

\section{3. Описание подложек}

Работа проводилась с использованием подложек Prefabricated OFET Test Chips компании Osilla, изготовленных на основе высоколегированной кремниевой пластины с предварительно нанесенными металлическими электродами. Ширина канала -1 мм, длина канала 30 мкм, толщина изолятора из оксида кремния -300 нм, емкость изолятора $1.09 \cdot 10^{-8} \Phi / \mathrm{cm}^{2}$. Пример применения подложек приводится в работе [16]. Поскольку оксид кремния образует акцепторные ловушечные состояния, то производитель требует силанизации подложек, указывая на невозможность в противном случае исследовать электронную подвижность.

Наличие в оксиде кремния множества акцепторных вакансий и их влияние на работу транзистора - хорошо изученная тема, например в работе [17]. Известно, что такие состояния способны привести к увеличению $V_{\text {th }}[18]$, а в работе [14] показано, что ненулевое напряжение $V_{\text {on }}$ свидетельствует о наличии заряженных ловушечных состояний на интерфейсе с диэлектриком.

Проведение работы без силанизации возможно, но будет сопряжено с очень большим напряжением включения $V_{\mathrm{TH}}$. Так, в работе [19] показано изменение $V_{\text {th }}$ c 41 и 13 В для электронов и дырок, соответственно, в случае чистого оксида кремния до 27 и -11 В после обработки гексаметилдисилазаном (Hexamethyldisilazane, HMDS), что показывает значительно меньший захват электронов в ловушки. Сравнение шести разных силанов проводится в работе [20], где наилучшая подвижность электронов регистрируется с HMDS $\left(V_{\text {on-e }}=V_{\text {on-h }}=-5 \mathrm{~B}\right)$, 
a наилучшая подвижность дырок и одновременно худшая у электронов - с додецилтрихлоросиланом (Dodecyltrichlorosilane). Статья дополнительно показывает, что силанизация - вовсе не необходимый этап для исследования образцов $\mathrm{HK} \mathrm{PbS}$, но выбор вещества для силанизации влияет на результаты и может привести к значительному завышению мобильности.

\section{4. Исследование подвижности носителей зарядов}

В работе использовались $\mathrm{HK} \mathrm{PbS}$ диаметром $3.4 \mathrm{HM}$ (пик поглощения 895 нм), изготовленные методом металлорганического синтеза [21]. Пленки $\mathrm{HK} \mathrm{PbS}$ наносились методом спин-коатинга с последующей заменой исходных лигандов олеиновой кислоты на EDT и TBAI. Для этого $\mathrm{HK}$ PbS переводились в раствор октана, TBAI растворялся в метаноле (15 мг ТВАІ на 1 мл метанола), a EDT растворялся в ацетонитриле (0.1\% раствор). После этого раствор $\mathrm{HK} \mathrm{PbS}$ наносился на вращающуюся подложку методом спин-коатера, для замены на слой $\mathrm{HK} \mathrm{PbS}$ на 30 с наносился раствор соответствующих лигандов и следом промывался ацетонитрилом - он также наносился на 30 с и затем подложка раскручивалась опять. Вся процедура повторялась 3 раза для получения пленки толщиной 70-80 нм.

Предварительно проводилась силанизация поверхности подложек при помощи HMDS, для чего подложка 20 мин выдерживалась в растворе HMDS (0.1 мл HDMS на 4 мл циклогексана), после чего промывалась циклогексаном.

Исследование пленках $\mathrm{HK} \mathrm{PbS}-\mathrm{TBAI}$ сперва проводилось на воздухе. На выходных характеристиках насыщение не наблюдается, и работа идет в линейном режиме во всем диапазоне напряжений (рис. 1). В области положительного смещения при увеличении напряжения на затворе ток заметно падает, в области отрицатель-

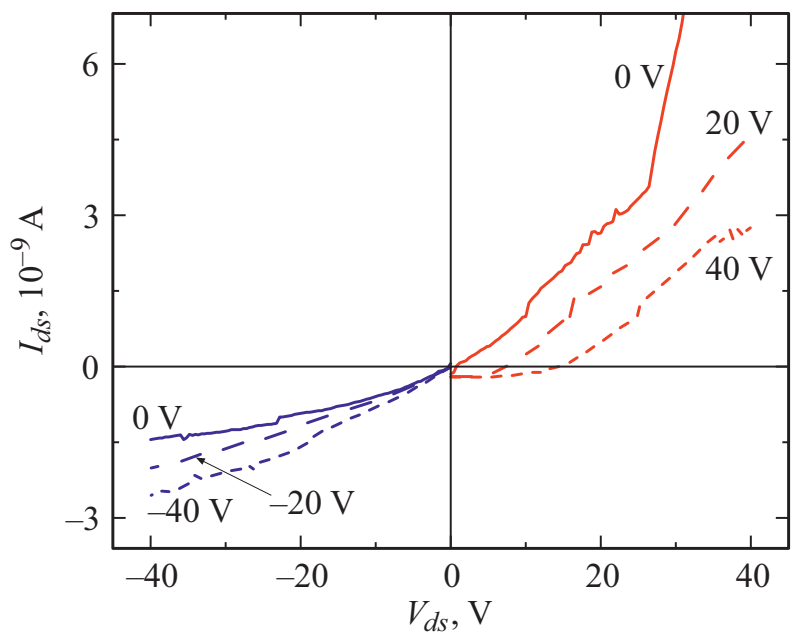

Рис. 1. Выходные характеристики пленки $\mathrm{HK} \mathrm{PbS}-\mathrm{TBAI}$ на воздухе.

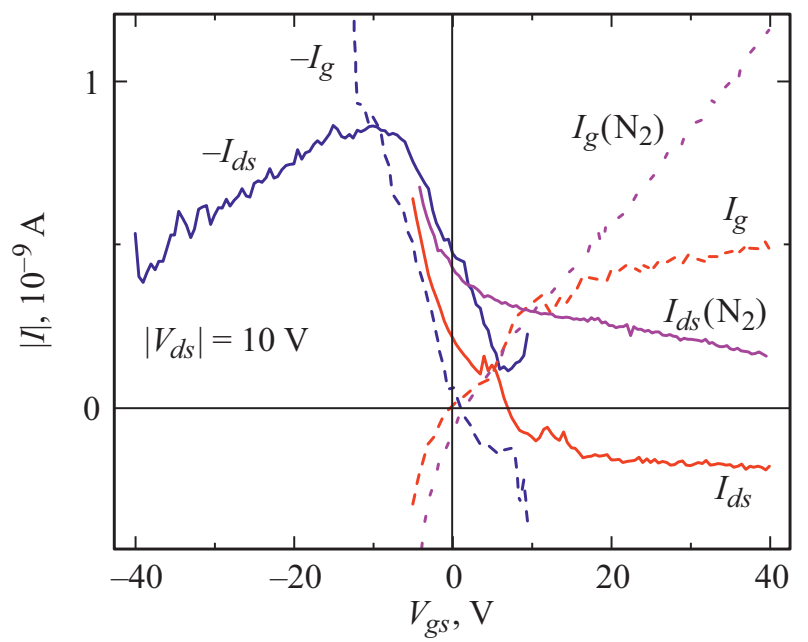

Рис. 2. Передаточная характеристика пленки $\mathrm{HK} \mathrm{PbS}-\mathrm{TBAI}$ на воздухе и в инертной среде $\left(\mathrm{N}_{2}\right) . V_{\mathrm{th}-\mathrm{h}}=6.5 \mathrm{~B}$. Подвижность равна $3.0 \cdot 10^{-5} \mathrm{~cm}^{2} /(\mathrm{B} \cdot \mathrm{c})$. (Цветной вариант рисунка представлен в электронной версии статьи).

ного - немного увеличивается, т.е. в обоих случаях демонстрируются признаки дырочной проводимости. Асимметрия выходных характеристик, когда даже при нулевом напряжении на затворе ток в положительной части выше, вызвана влиянием поля затвора на канал и на контакт стока, и пропадает при отключении затвора.

Передаточные характеристики были получены при напряжении $V_{d s}=10 \mathrm{~B}$ (рис. 2). На $n$-канальной характеристике участков роста не наблюдается, ток падает и уходит в отрицательную сторону. Ключом к пониманию отрицательного тока при положительном напряжении на стоке является ток, регистрируемый в затворе, - он практически зеркален току в канале, и при этом на порядок превышает возможный ток утечки. Если исключать вариант пробоя изолятора (при котором ток линеен, гораздо выше и одинаков в обоих $p$ - и $n$-канальной характеристиках), то очевидно, что происходит зарядка емкости „канал-затвор“. При этом электроны заполняют акцепторные состояния (в оксиде кремния или иные акцепторные состояния), вызывая зеркальный приток положительного заряда в затвор по другую сторону изолятора. При этом ток в канале отсутствует, за исключением тока заряда емкости.

На $p$-канальной характеристике наблюдается линейный участок роста, начинающийся уже при положительном смещении $V_{\text {th-h }}=6.5 \mathrm{~B}$, выходящий на насыщение после $V_{g s}=-6 \mathrm{~B}$ и начинающий спадать после $-15 \mathrm{~B}$. При этом значении напряжения ток, регистрируемый в контакте затвора, превышает ток в канале, и, судя по его знаку, затвор стремительно заряжается отрицательно. Вероятнее всего, после достижения насыщения на границе слоя НК и изолятора скапливается значительный положительный заряд, препятствующий влиянию затвора и протеканию тока. Это и отображается в виде уменьшения отрицательного тока в канале. При 


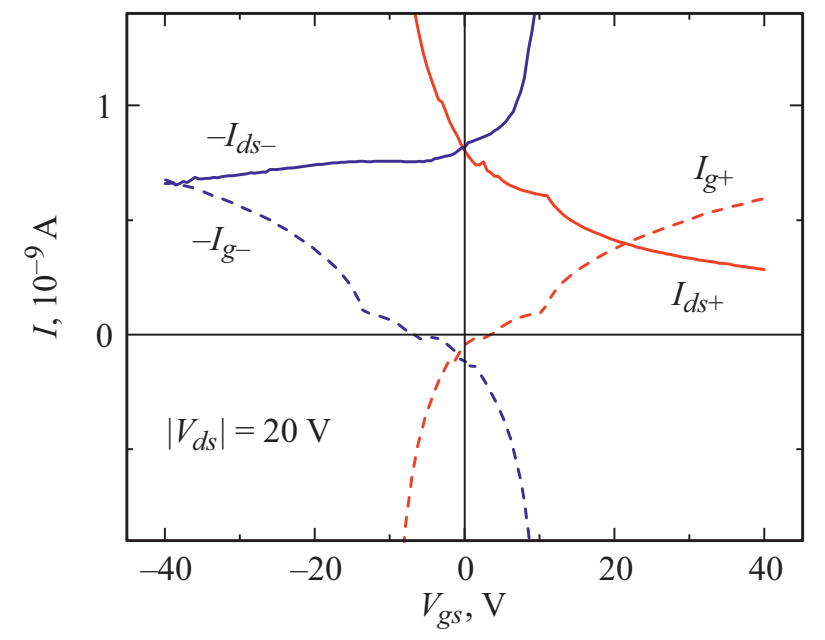

Рис. 3. Передаточная характеристика пленки $\mathrm{HK} \mathrm{PbS}-\mathrm{EDT}$ на воздухе.

повышенном напряжении $V_{d s}=20$ В линейный характер сохраняется до значения смещения $V_{g s}=-40 \mathrm{~B}$, это говорит о том, что выбранный участок не является точкой перегиба. Рассчитанная подвижность составила $3.0 \cdot 10^{-5} \mathrm{~cm}^{2} /(\mathrm{B} \cdot \mathrm{c})$, что находится в диапазоне литературных данных, где подвижность может составлять от $2 \cdot 10^{-7}$ [22] до $2 \cdot 10^{-3}$ [23], хотя это ниже более распространенного значения, составляющего $\sim 10^{-4} \mathrm{~cm}^{2} /(\mathrm{B} \cdot \mathrm{c})[24,25]$.

Перенос процедуры нанесения и измерений в инертную среду перчаточного бокса к существенному изменению картины не привел, за одним заметным исключением: на $n$-канальной характеристике ток канала $I_{\mathrm{SD}}$ хотя и уменьшается при увеличении смещении, но не уходит в отрицательную часть, а ток затвора теперь растет линейно (см. рис. 2). Таким образом, теперь „зеркальная“ зарядка канала и затвора не происходит, и спад тока может уже означать просто отсутствие электронной проводимости, хотя это и не исключает присутствия эффекта экранирования, не позволяющего зарегистрировать подвижность электронов.

Выходные характеристики, полученные для пленок $\mathrm{HK} \mathrm{PbS}-\mathrm{EDT}$, показывают независимость от напряжения на затворе. Практически то же самое можно сказать про передаточные характеристики, показанные на рис. 3, - ток в канале уменьшается независимо от полярности напряжения на затворе, а ток через затвор $I_{g}$ сопоставим с величиной тока через канал $I_{s d}$. Добавим, что при низкой скорости прироста напряжения на затворе ток падает до минимума уже при низких напряжениях, т.е. канал оказывается изолирован от влияния потенциала затвора, и это опять можно объяснить образованием индуцированной полем затвора области заряда, расположенной на границе канала с диэлектрическим слоем. Но если такое поведение в области n-канальной проводимости можно объяснить акцепторными состояниями оксида кремния, то поведение в области $p$-канальной проводимости объяснимо только при образовании ловушечных донорных состояний в самом слое НК.

В воздушной среде образец $\mathrm{HK} \mathrm{PbS}-\mathrm{EDT}$ уже на следующий день не позволял получить никакие выходные характеристики, в то время как образец с TBAI еще несколько дней демонстрировал передаточные характеристики, хотя и с ухудшающимися параметрами.

\section{5. Исследование на свету}

$\mathrm{HK} \mathrm{PbS}-\mathrm{EDT}$ обычно используется как слой, блокирующий электроны, и делается небольшой толщины (чтобы блокировать электроны, но не вносить излишних резистивных потерь). Поэтому отсутствие электронной проводимости не является проблемой. В слое $\mathrm{HK} \mathrm{PbS}-\mathrm{TBAI}$, напротив, происходит образование электронно-дырочных пар и их разделение полем, поэтому должны наблюдаться оба типа проводимости. Процессы в слоях НК на свету отличаются от процессов в темноте тем, что в первом случае образуется больше носителей зарядов, а ловушки - если они относительно не глубокие - активируются и высвобождают заряды. В этом случае появление дополнительных фотоактивированных носителей может показать, что предположения относительно отсутствия электронной или дырочной проводимости преждевременны. Для этого мы проверили поведение образцов, обработанных EDT и TBAI, при освещении симулятором солнечного излучения на основе ксеноновой лампы [26].

Интенсивность излучения в симуляторе составляет $100 \mathrm{MBT} / \mathrm{cm}^{2}$ (спектр АM1.5G), симулятор использовался совместно со светофильтром ЖС11 для удаления из спектра излучения с длиной волны короче $400 \mathrm{HM}$, так как оно быстро повреждало образцы. Для ослабления светового потока мы использовали стеклянный нейтральный светофильтр НС7 и две фотометрические сетки с пропусканием 25 и 50\%. В итоге, последовательно добавляя светофильтр НС7 и сетки, мы получали освещенность $87,32,8$ и 4\% от исходной интенсивности симулятора при исследовании пленок $\mathrm{HK}$ PbS-TBAI и 87, 32 и 4\% при исследовании пленок HK PbS-EDT.

Анализ изменения наклона передаточной характеристики образца $\mathrm{HK} \mathrm{PbS}-\mathrm{TBAI}$ в области $p$-канальной проводимости при увеличении освещения (см. рис. 4) показывает, что подвижность выросла в 4 раза (с $1.37 \cdot 10^{-4}$ до $5.39 \cdot 10^{-4} \mathrm{~cm}^{2} /(\mathrm{B} \cdot \mathrm{c})$ ), а ток в 3.2 раза. Таким образом, поскольку проводимость определяется как $\sigma=q n \mu$, где $q$ - элементарный заряд, $n-$ концентрация носителей и $\mu-$ подвижность, основной вклад в увеличение проводимости внес рост подвижности.

В области $n$-канальной проводимости ток спадает при росте напряжения на затворе при всех уровнях освещенности, но общий уровень тока растет вместе с освещенностью, равно как и ток затвора, при этом ток затвора ниже тока в канале в 2-3 раза, т. е. зарядка 
ловушек идет, но на проводимость в канале это влияет гораздо слабее, чем в темноте.

В образцах $\mathrm{HK} \mathrm{PbS}-\mathrm{EDT}$ ток через канал возрастает в обеих полярностях одинаково с ростом освещенности (рис. 5), на передаточных характеристиках реакция на затвор не наблюдается, а ток затвора при изменении освещенности практически не растет, т.е. новых вакансий при освещении не образуется.

Обратим внимание на две особенности. Во-первых, поскольку ток в канале одинаковый в обе стороны, и можно полагать, что канал экранирован и свободные носители заряда не испытывают влияние затвора, то можно считать, что в обеих полярностях принимают участие одни и те же носители заряда (но идентифицировать их тут невозможно). Во-вторых, интересно то, что

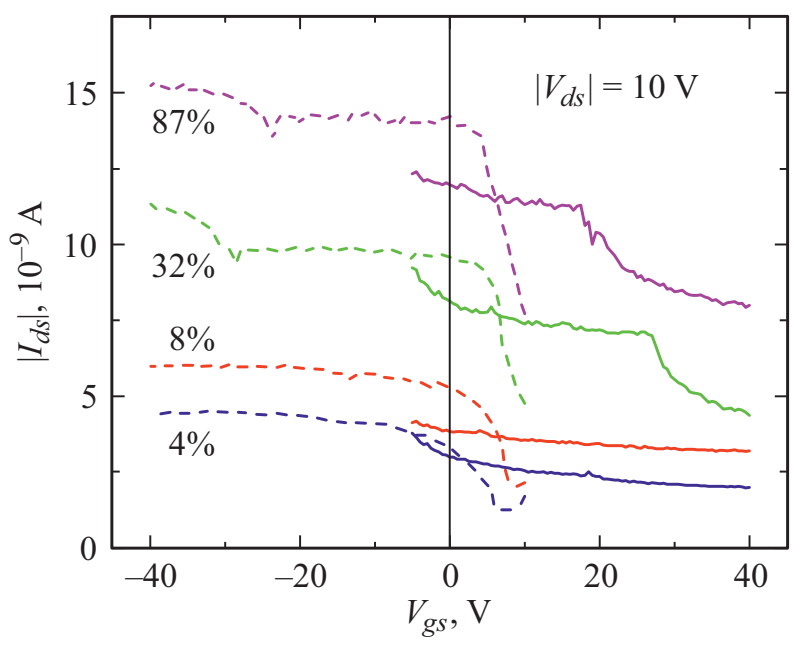

Рис. 4. Передаточные характеристики образцов НК $\mathrm{PbS}-\mathrm{TBAI}$. Ток затвора при $V_{G S}=-40 \mathrm{~B}$ во всем диапазоне освещенности составил 0.9 нА и поэтому не показан. При $V_{G S}=40 \mathrm{~B}$ ток затвора меняется соответственно изменению освещенности от 0.8 до 2.8 нА.

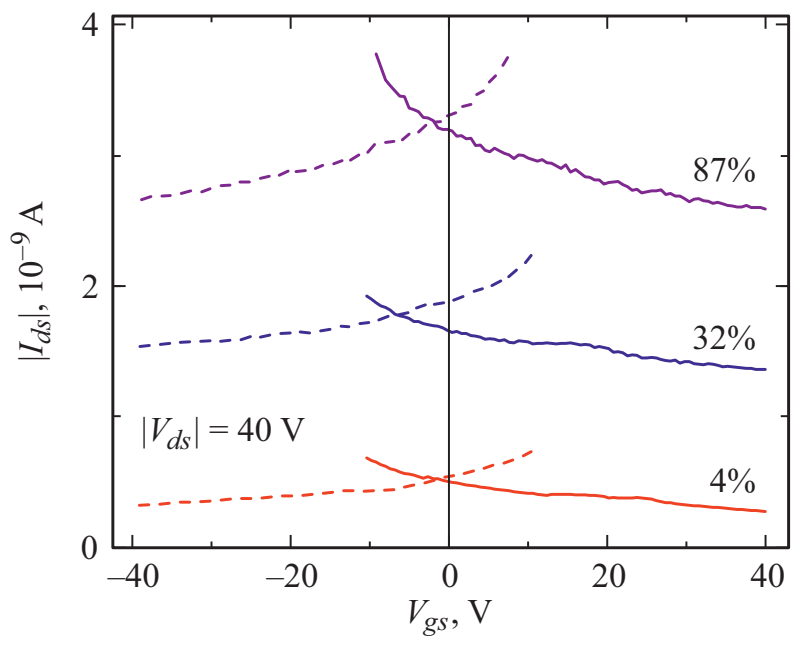

Рис. 5. Передаточные характеристики образцов $\mathrm{HK}$ PbS-EDT при освещении.

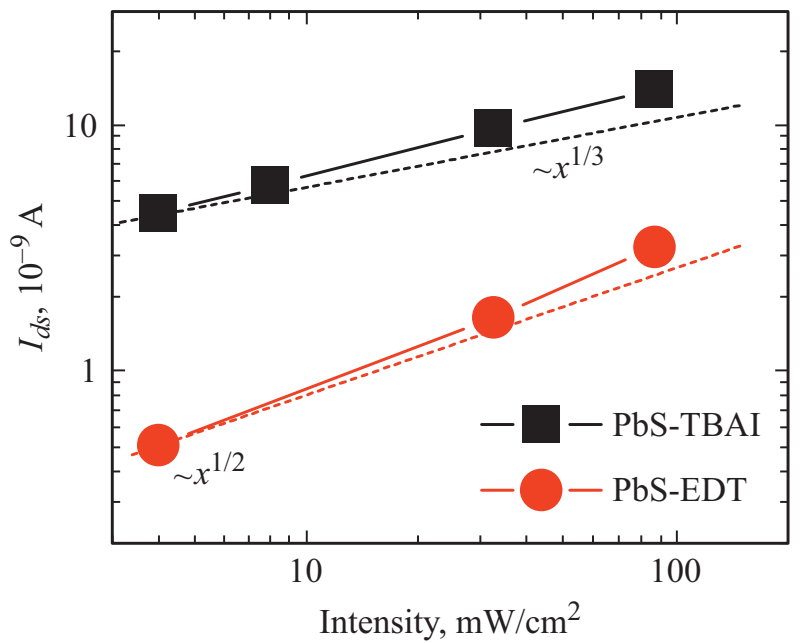

Рис. 6. Зависимость тока образцов $\mathrm{HK} \mathrm{PbS}-\mathrm{TBAI} / \mathrm{EDT}$ от освещенности. Штриховые линии показывают наклон степенной функции с показателем степени $1 / 2$ и $1 / 3$.

освещение образцов с EDT даже с 4\% интенсивностью настолько улучшает проводимость, что ток в канале становится много выше тока зарядки затворной емкости, при этом последний при росте освещенности изменяется не так существенно - от 0.26 до $0.5 \mathrm{HA} \mathrm{(в} \mathrm{темноте}$ он составлял $0.1 \mathrm{HA}$ ). Вероятно, что при освещении подвижность носителей заряда заметно вырастает, и они больше участвуют в образовании тока в канале, нежели попадают в ловушки, таким образом не вызывая существенного заряда емкости полевого транзистора.

Теперь рассмотрим зависимость проводимости от освещенности. В случае полупроводника такая зависимость описывается степенным законом, и в идеале степень равна 1, но из-за влияния ловушек показатель обычно уменьшается до целых долей единицы [27]. В случае образца $\mathrm{PbS}-\mathrm{EDT}$ ток, а следовательно, и проводимость увеличиваются пропорционально степени 0.60 , что близко к $1 / 2$ (см. рис. 6). В случае образа PbS-TBAI показатель степени составляет 0.37 , что составляет $\sim 1 / 3$. Это является косвенным подтверждением того, что $\mathrm{HK} \mathrm{PbS}-\mathrm{EDT}$ обладают окисленной поверхностью, поскольку степень $1 / 3$ означает поверхностно-доминирующую проводимость, а $1 / 2$ - проводимость твердого тела [28], и переход от зависимости $1 / 3$ к 1/2 происходит из-за окисления поверхности НК, при котором появляющиеся поверхностные ловушки иммобилизируют заряды фототока.

Всего при освещенности проводимость слоя НК $\mathrm{PbS}$-TBAI выросла в 14 раз (в темноте ток составил $\left.10^{-9} \mathrm{~A}\right)$, что близко к показателям из статьи [6], где проводимость выросла в 30-60 раз при освещенности $300 \mathrm{MB} / \mathrm{cm}^{2}$, так как при увеличении освещенности в 3 раза проводимость бы выросла в итоге в 24 раза. В случае $\mathrm{PbS}-\mathrm{EDT}$ проводимость выросла сильнее, но ток в целом был меньше. 


\section{3. Обсуждение результатов}

У образцов $\mathrm{HK}$ PbS-TBAI измерена подвижность дырок, пороговое напряжение $V_{\text {TH }}$ составляет $\sim 5-10 \mathrm{~B}$ и говорит о небольшой поляризации диэлектрика или интерфейса с ним, также просматривается разница в образцах, изготовленных без доступа воздуха и с ним. Образцы $\mathrm{HK} \mathrm{PbS}-\mathrm{EDT}$, независимо от условий изготовления, из-за образования ловушечных состояний не позволили оценить тип проводимости. В то же время поляризация перестает быть доминирующим процессом уже при невысоком уровне засветки. Также в эксперименте с освещением показано, что на фотопроводимость образцов $\mathrm{HK} \mathrm{PbS}-\mathrm{EDT}$ существенно влияют поверхностные ловушечные состояния.

В целом работа подтверждает выводы других исследователей, что слой $\mathrm{HK} \mathrm{PbS}-\mathrm{EDT}$ является слабым местом солнечных элементов рассматриваемой структуры [3]. По этой причине замену лигандов EDT желательно проводить в растворе, а не после нанесения [29]. Рекомендуемой заменой EDT являются галогенидные лиганды (хлор и фтор) из-за меньшей летучести, токсичности и большей стабильности [30].

Обозначим причины, сделавшие невозможным анализ подвижности носителей заряда в образцах. Так, это могли быть акцепторные ловушки в слое изолятора, экранирующие поле затвора и мешающие регистрировать подвижность электронов. Но поверхность подложки была обработана таким же силаном HMDS, как и в исследовании [20], где напряжение включения $V_{\text {th }}$ составило $\sim 30$ В. Поэтому более вероятной причиной являются ловушечные состояния на поверхности самих НК или на интерфейсе с поверхностью изолятора, также вызывающие эффект экранирования. При этом, если в случае НК PBS-TBAI это акцепторные состояния, возможно возникшие из-за окисления поверхности, то в случае $\mathrm{HK} \mathrm{PbS}-\mathrm{EDT}$ добавляются ловушечные донорные состояния. Это очень нежелательный эффект, поскольку слой $\mathrm{HK} \mathrm{PbS}-\mathrm{EDT}$ применяется как слой транспорта дырок, и это может вызывать ухудшение в работе устройств на их основе.

Возможно, что кроме ухудшения пассивации поверхности (что ведет к окислению НК и легированию $p$-типа) образуются и дополнительные ловушки из-за непосредственно EDT, особенно если он плохо вымывается из слоя НК [31]. Исследование в работе [6] показывает, что проблема может происходить от самого EDT, который разлагается на воздухе. Версия некачественной замены рассматривалась отдельно, процедура замены лигандов была проанализирована и нарушений обнаружено не было. Поскольку неполная замена лигандов ведет к завышенному сопротивлению, то мы сравнили удельное сопротивление образцов. Оно составило $12-25$ кОм · м и находится в диапазоне опубликованных в литературе данных 5-40 кОм · м $[5,30,32]$.

Чтобы исключить вариант того, что подложка сама по себе не допускает регистрации электронной прово- димости, на ней была измерена подвижность в слое $\mathrm{HK} \mathrm{ZnO}$ (производство Sigma-Aldrich) на воздухе. Измерение показало электронную проводимость и довольно низкое значение $V_{\text {th }}(<10 \mathrm{~B})$. Это подтверждает то, что подложку с изолятором из оксида кремния можно использовать для анализа электронной проводимости при проведении силанизации.

\section{4. Заключение}

В работе показано, что для $\mathrm{HK} \mathrm{PbS}$ на воздухе преобладающим типом является дырочная проводимость для обоих типов лигандов, даже для HK PbS-TBAI, которые должны были показать электронную проводимость на воздухе. Очевидно, что описание работы СЭ на воздухе будет заметно отличаться от инкапсулированных элементов. При анализе передаточных характеристик особое внимание уделено току затвора, показывающему накопление заряда в канале. Показано, что в случае пленок $\mathrm{HK} \mathrm{PbS}-\mathrm{EDT}$ образуются ловушечные донорные состояния, затрудняющих дырочную проводимость. Исследование влияния освещения на фотопроводимость показало различающийся механизм проводимости в слоях $\mathrm{HK} \mathrm{PbS}$ с лигандами TBAI и EDT, предположительно, из-за окисления поверхности последних.

\section{Финансирование работы}

Работа выполнена при поддержке Российского научного фонда (проект № 19-13-00332).

\section{Конфоликт интересов}

Авторы заявляют, что у них нет конфликта интересов.

\section{Список литературы}

[1] Y. Liu, M. Gibbs, J. Puthussery, S. Gaik, R. Ihly, H.W. Hillhouse, M. Law. Nano Lett., 10, 1960 (2010).

[2] P.R. Brown, D. Kim, R.R. Lunt, N. Zhao, M.G. Bawendi, J.C. Grossman, V. Bulović. ACS Nano, 8, 5863 (2014).

[3] W. Gao, G. Zhai, C. Zhang, Z. Shao, L. Zheng, Y. Zhang, Y. Yang, X. Li, X. Liu, B. Xu. RSC Adv., 8, 15149 (2018).

[4] A.P. Litvin, P.S. Parfenov, E.V. Ushakova, A.V. Fedorov, M.V. Artemyev, A.V. Prudnikau, V.V. Golubkov, A.V. Baranov. J. Phys. Chem. C, 117, 12318 (2013).

[5] I.D. Skurlov, I.G. Korzhenevskii, A.S. Mudrak, A. Dubavik, S.A. Cherevkov, P.S. Parfenov, X. Zhang, A.V. Fedorov, A.P. Litvin, A.V. Baranov. Materials (Basel), 12, 3219 (2019).

[6] J.M. Luther, M. Law, Q. Song, C.L. Perkins, M.C. Beard, A.J. Nozik. ACS Nano, 2, 271 (2008).

[7] A.A. Babaev, P.S. Parfenov, D.A. Onishchuk, A. Dubavik, S.A. Cherevkov, A.V. Rybin, M.A. Baranov, A.V. Baranov, A.P. Litvin, A.V. Fedorov. Materials (Basel), 12, 4221 (2019).

[8] I.G. Korzhenevskii, D.A. Onishchuk, A.A. Babaev, A. Dubavik, P.S. Parfenov, A.P. Litvin. Semiconductors, 53, 1946 (2019). 
[9] L. Hu, A. Mandelis, X. Lan, A. Melnikov, S. Hoogland, E.H. Sargent. Sol. Energy Mater. Sol. Cells, 155, 155 (2016).

[10] X. Yang, L. Hu, H. Deng, K. Qiao, C. Hu, Z. Liu, S. Yuan, J. Khan, D. Li, J. Tang, H. Song, C. Cheng. Nano-Micro Lett., 9, 24 (2017).

[11] H. Wang, S. Yang, Y. Wang, J. Xu, Y. Huang, W. Li, B. He, S. Muhammad, Y. Jiang, Y. Tang, B. Zou. Org. Electron., 42, 309 (2017).

[12] V. Podzorov. MRS Bull., 38, 15 (2013).

[13] J. Chang, Z. Lin, C. Zhang, Y. Hao. In: Differ. Types Field-Effect Transistors-Theory Appl. (InTech, 2017).

[14] V. Podzorov, M.E. Gershenson, C. Kloc, R. Zeis, E. Bucher. Appl. Phys. Lett., 84, 3301 (2004).

[15] H.H. Choi, K. Cho, C.D. Frisbie, H. Sirringhaus, V. Podzorov. Nature Materials, 17, 2 (2018).

[16] C. Pérez-Fuster, J.V. Lidón-Roger, L. Contat-Rodrigo, E. García-Breijo. J. Sensors, 2018, 1 (2018).

[17] Y.Y. Illarionov, G. Rzepa, M. Waltl, T. Knobloch, A. Grill, M.M. Furchi, T. Mueller, T. Grasser. 2D Mater., 3, 035004 (2016).

[18] A. Daus, C. Vogt, N. Munzenrieder, L. Petti, S. Knobelspies, G. Cantarella, M. Luisier, G.A. Salvatore, G. Troster. IEEE Trans. Electron Dev., 64, 2789 (2017).

[19] M.I. Nugraha, R. Häusermann, S.Z. Bisri, H. Matsui, M. Sytnyk, W. Heiss, J. Takeya, M.A. Loi. Adv. Mater., 27, 2107 (2015).

[20] M.I. Nugraha, H. Matsui, S.Z. Bisri, M. Sytnyk, W. Heiss, M.A. Loi, J. Takeya. APL Mater., 4, 116105 (2016).

[21] M.A. Hines, G.D. Scholes. Adv. Mater., 15, 1844 (2003).

[22] M.J. Speirs, D.N. Dirin, M. Abdu-Aguye, D.M. Balazs, M.V. Kovalenko, M.A. Loi. Energy Environ. Sci., 9, 2916 (2016).

[23] S.Z. Bisri, C. Piliego, M. Yarema, W. Heiss, M.A. Loi. Adv. Mater., 25, 4309 (2013).

[24] K. Lu, Y. Wang, Z. Liu, L. Han, G. Shi, H. Fang, J. Chen, X. Ye, S. Chen, F. Yang, A.G. Shulga, T. Wu, M. Gu, S. Zhou, J. Fan, M.A. Loi, W. Ma. Adv. Mater., 30, 1707572 (2018).

[25] Q. Lin, H. J. Yun, W. Liu, H.-J. Song, N.S. Makarov, O. Isaienko, T. Nakotte, G. Chen, H. Luo, V.I. Klimov, J.M. Pietryga. J. Am. Chem. Soc., 139, 6644 (2017).

[26] A.P. Litvin, I.D. Skurlov, I.G. Korzhenevskii, A. Dubavik, S.A. Cherevkov, A.V. Sokolova, P.S. Parfenov, D.A. Onishchuk, V.V. Zakharov, E.V. Ushakova, X. Zhang, A.V. Fedorov, A.V. Baranov. J. Phys. Chem. C, 123, 3115 (2019).

[27] Y. Aoyagi, K. Masuda, S. Namba. J. Appl. Phys., 43, 249 (1972).

[28] P. Irkhin, H. Najafov, V. Podzorov. Sci. Rep., 5, 15323 (2015).

[29] J.L. Peters, J.C. van der Bok, J.P. Hofmann, D. Vanmaekelbergh. Chem. Mater., 31, 5808 (2019).

[30] D. Bederak, D.M. Balazs, N.V. Sukharevska, A.G. Shulga, M. Abdu-Aguye, D.N. Dirin, M.V. Kovalenko, M.A. Loi. ACS Appl. Nano Mater., 1, 6882 (2018).

[31] R. D. Septianto, L. Liu, F. Iskandar, N. Matsushita, Y. Iwasa, S.Z. Bisri. NPG Asia Mater., 12, 33 (2020).

[32] K.S. Jeong, J. Tang, H. Liu, J. Kim, A.W. Schaefer, K. Kemp, L. Levina, X. Wang, S. Hoogland, R. Debnath, L. Brzozowski, E.H. Sargent, J.B. Asbury. ACS Nano, 6, 89 (2012).

\section{Study of charge carrier mobility in PBS nanocrystal layers using field-effect transistors}

\section{P.S. Parfenov, N.V. Bukhryakov, D.A. Onishchuk, A.A. Babaev, A.V. Sokolova, A.P. Litvin}

ITMO University, Center of Information

Optical Technologies,

197101 St. Petersburg, Russia

Abstract The field-effect transistor method is used to study the mobility of charge carriers in layers of lead sulfide nanocrystals with ligands of tetrabutylammonium iodide and 1,2-ethanedithiol used to create solar cells. The difference between the operating of a transistor in ambient air and in an inert atmosphere is demonstrated. It is shown that, in the ambient air, the processes of charging nanocrystals are activated when current flows, and the influence of the polarization of the interface of nanocrystals and the insulator on the measurement of the mobility is analyzed. Different reactions of the layers with ligands to light have been demonstrated, showing a significant oxidation of the surface of nanocrystals treated with 1,2-ethanedithiol.

Редактор Г.А. Оганесян 